\title{
Storia di un'assenza. Le fotografie della zona libera della val Chisone
}

Mira, Toni Rovatti

ISSN: 2282-4979

DOI: 10.12977/ereview89

Questo articolo è stato sottoposto a un processo di double blind peer review

Il saggio studia la rappresentazione fotografica della Resistenza italiana negli scatti del partigiano Ettore Serafino e si sofferma in particolare sulle immagini di un evento specifico: la costituzione della zona libera della val Chisone, in provincia di Torino, dove Serafino fu al comando di una formazione autonoma. A partire dall'esame di un caso di studio, l'articolo cerca di affrontare il problema della rappresentazione fotografica delle zone libere del 1944 in Italia. L'obiettivo di fondo è analizzare i meccanismi di formazione della memoria fotografica del passato; essa non ha una sola causa, ma che è piuttosto il frutto dell'interazione di numerose variabili: locali e sovra locali, individuali e collettive, private e pubbliche, politiche, sociali e culturali, relative alle forme di formazione e stratificazione della memoria.

The paper studies the photographic representation of the Italian Resistance in the shots of the partisan Ettore Serafino and it focuses in particular on the images of a specific event: the establishment of the "free zone" in the Val Chisone (in the north of Italia, near Turin), where Serafino was the commander of an "autonomous" partisan formation. Analysing a case study, the paper address the issue of photographic representation of the "free zones" in Italy during 1944. The principal aim is to examine the mechanisms of formation of the photographic memory of the past; this memory does not have a single cause, but rather it is the result of the interaction of a number of variables: local and supra local, individual and collective, private and public, political, social and cultural, and other factors depending on process of stratification of memory.

Questo saggio nasce da una ricerca in corso sulla rappresentazione fotografica della Resistenza italiana negli scatti del partigiano Ettore Serafino e si sofferma in particolare sulle immagini di un evento specifico: la costituzione della zona libera della val Chisone, in provincia di Torino, dove Serafino fu al comando di 
una formazione autonoma'. A partire dall'esame di un caso di studio, l'articolo cercherà di affrontare il problema della rappresentazione fotografica delle zone libere del 1944 in Italia; trattandosi di uno studio in corso, esso fornirà i primi risultati dell'indagine e presenterà alcune ipotesi di lavoro². L'obiettivo di fondo è analizzare i meccanismi di formazione della memoria fotografica, che non ha una sola causa, ma che è piuttosto il frutto dell'interazione di numerose variabili: politiche, sociali e culturali, locali e sovra locali, individuali e collettive, relative al genere, al rapporto tra passato e presente, alle forme di costruzione e stratificazione della memoria. Concentrare l'attenzione su un singolo caso permetterà dunque di metterne in luce alcune e di cominciare a evidenziare i modi in cui sono tra loro interconnesse.

La ricerca sulle fotografie della zona libera della val Chisone è iniziata da una constatazione: tra i circa trecento scatti del fondo fotografico di Serafino relativi al periodo partigiano (per lui iniziato precocemente già l'8 settembre 1943) non è presente nessuna immagine delle settimane di autogoverno partigiano³. Ma perché? Il tentativo di rispondere a tale interrogativo mi ha spinto anzitutto ad approfondire l'analisi delle caratteristiche del fondo personale, della Resistenza nella vallata torinese e delle sue fotografie: per spiegare l'assenza ho dunque cercato di formulare alcune ipotesi legate alle vicende di Serafino e alla storia della Resistenza in valle. In un secondo momento ho cercato di allargare l'osservazione ad altri contesti locali, per provare a individuare peculiarità e analogie tra l'assenza di fotografie della zona libera della val Chisone e la rappresentazione fotografica

1 Devo ringraziare la famiglia Serafino per l'accesso al fondo fotografico e ad alcuni documenti cartacei. In particolare Adolfo Serafino mi ha aiutato a scavare nelle carte di suo papà e ha attinto diverse informazioni dai ricordi personali e familiari. Un contributo prezioso nell'individuazione di fondi fotografici piemontesi mi è venuto da Andrea D'Arrigo, archivista dell'Istituto Piemontese per la storia della Resistenza e della società contemporanea Giorgio Agosti (d'ora in avanti Istoreto).

2 Solo recentemente gli storici hanno iniziato a rivolgere la propria attenzione allo studio della fotografia della guerra civile italiana. Nonostante alcuni contributi fondamentali [Mignemi 1995 e 2000; De Luna e Mignemi (eds.) 1997; Mignemi e Solaro (eds.) 2005], essa rappresenta ancora un campo di indagine aperto. In generale la storiografia, non solo italiana, sconta un enorme ritardo nell'uso delle fotografie come fonte [Freund 1976; Linguaggio e fotografia 1977; Fotografia e stile 1978; Ortoleva 1983; Sega 1988; Zannier 1978 e 1986; De Luna 1993; Burke 2002; Mignemi 2003; D’Autilia 2005; Clarke 2009], anche se alcuni classici contengono fondamentali considerazioni teoriche e metodologiche [Benjamin 1966; Bourdieu (ed.) 1972; Sontag 1978; Barthes 1980].

3 Dall'inizio dell'estate del 1944 Serafino combatté proprio in val Chisone, e tra i primi di giugno e l'inizio di agosto l'alta valle passò sotto il totale controllo partigiano. La valle va dunque annoverata tra le zone libere del 1944, pur non essendo presente negli elenchi ufficiali. Tra fine maggio e giugno i fascisti e i tedeschi lasciarono quella parte del territorio alpino per attestarsi a valle del comune di Perosa Argentina, in attesa di lanciare la grande offensiva denominata Nachtigall; tutti i centri fino a Sestrière furono così governati per circa due mesi dai partigiani. 
di esperienze simili nell'Italia centro-settentrionale durante il $1944^{4}$.

\section{Ettore Serafino e il suo fondo fotografico}

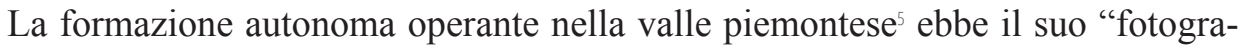
fo ufficiale": Ettore Serafino. Non un fotografo professionista, come raramente capitò ai gruppi partigiani durante la guerra ${ }^{6}$. Piuttosto, Serafino era un ventenne avviato alla carriera forense con la passione della fotografia trasmessagli dalla famiglia ${ }^{7}$, che, poco prima della guerra, gli aveva regalato una macchina portatile, una Kodak retina II con obiettivo da 50 millimetri. Ma chi era Ettore e come si trovò a combattere contro i nazi-fascisti in val Chisone?

Nato nel 1918 a Rivarolo Canavese, un piccolo centro della provincia di Torino dove lavorava in quel momento il padre, ingegnere del settore conciario, Ettore Serafino fu chiamato alle armi nel 1939 nel $3^{\circ}$ Reggimento alpini col grado di sottotenente di complemento, mentre frequentala la Facoltà di Giurisprudenza a Torino. Con l'ingresso in guerra dell'Italia, fu mandato a combattere prima nel fronte occidentale poi in quello balcanico. Tornato in Italia, nell'estate del 1943 completò gli studi giuridici, ottenne il grado di capitano di complemento e fu assegnato alla Scuola militare di alpinismo di Aosta, dove si trovò in servizio al momento dell'Armistizio ${ }^{8}$.

È lo stesso Serafino a raccontare il suo 8 settembre [Serafino 2001, 98-100]:

a un tratto le strade e le piazze furon piene di voci, di grida, non si comprendeva se di stupore, di gioia, di paura di esultanza o di sconforto.

Dalle porte spalancate dei bar, e sinanco delle case, usciva il gracidare di radio a

4 Ho limitato la ricerca alle fotografie partigiane, tralasciando gli scatti realizzati da fotografi al seguito delle truppe naziste e fasciste. Ciò che mi interessa, infatti, è decifrare la rappresentazione che i partigiani costruirono della loro guerra.

5 Nota successivamente col nome di $1^{\text {a }}$ Divisione Alpina Autonoma Val Chisone, poi con quello di $44^{\text {a Divisione Adolfo }}$ Serafino.

6 Fu il caso di Felice De Cavero, partigiano nelle Langhe e curatore di una delle prime mostre sulla Resistenza nel dopoguerra, di Luciano Giachetti, che con il cugino e compagno di lotta Adriano Ferraris fondò nel dopoguerra l'agenzia Fotocronisti Baita, di Aldo Corti, che ereditò dalla famiglia il negozio di barbiere e fotografo a Montefiorino nel modenese [Mignemi, 1995].

7 La passione fotografica familiare è attestata, oltre che dalla testimonianza del figlio Adolfo, dalla presenza nell'archivio personale di un album di famiglia realizzato dai genitori di Ettore.

8 Per ragioni di spazio non è possibile fornire in questa sede una puntuale ricostruzione biografica; per essa rimando all'intervista realizzata dall'Istoreto e registrata il 10 settembre 2008, che si può visionare online: http://www.metarchivi.it/ dett_documento.asp?id=11391\&tipo=VIDEO (ultima consultazione 27 maggio 2015). 
tutto volume. La guerra, la guerra terminata, un armistizio tra l'Italia e le potenze alleate era stato firmato: la resa, la sconfitta accettata e proclamata agitavano sentimenti di angoscia e incertezza [...]. Sia in lui, come nell'alpino che gli era assieme, come in altri che incontrava in strada e mutamente si interrogavano, era un agitarsi di speranze e di timori.

[...] Compresero che quanto prima avrebbero dovuto sciogliere la lor compagnia, il loro reparto, andarsene per la strada che ognuno avrebbe scelto, chi verso casa, se la si poteva raggiungere, chi altrove, magari senza una meta precisa, verso l'ignoto.

E così accadde dopo qualche giorno di inutile attesa. Se ne uscì dalla città, le vie già percorse da soldati tedeschi e risuonanti di secchi comandi, nella notte resa più buia dalla pioggia battente, trascinando con l'alpino Gianutin un mulo carico, per ogni evenienza possibile, d'armi, di viveri, di biada, di teli per rizzar la tenda, diretto, scavalcando colli e colli e attraversando valli e valli, al villaggio ove eran la casa, e i genitori del suo compagno di viaggio e di avventura, nella lontana Val Pellice?

Con Gianutin, cioè Giovanni, Serafino arrivò dunque a Bobbio Pellice verso la fine di settembre e divenne presto uno degli organizzatori delle prime bande partigiane in valle; nella primavera dell'anno seguente, anche a seguito di divergenze con i vertici partigiani della zona, si spostò in val Chisone dove divenne di fatto il vice di Marcellin: nel giugno del 1944 assunse il comando del Battaglione autonomo Monte Assietta, diventato poi Brigata, e in seguito della Brigata autonoma Monte Albergian. Nel marzo del 1945, nel quadro della riorganizzazione delle forze della Resistenza, diventò comandante della $44^{\mathrm{a}}$ Divisione Adolfo Serafino, intitolata al fratello caduto nel 1944.

Le fotografie di guerra di Ettore Serafino, conservate nell'archivio privato della famiglia ${ }^{10}$, riproducono il percorso del giovane che prima indossò la divisa dell'esercito e poi contribuì alla lotta partigiana". Il fondo fotografico si presenta così come organizzato dall'autore e comprende tre album con circa milleottocento im-

9 II volume raccoglie i ricordi dell'autore sulla guerra, inframmezzati da riflessioni religiose e poesie.

10 Sono in corso le pratiche di donazione delle immagini della guerra in copia digitale all'Istoreto.

11 Sulle fotografie di famiglia e sugli archivi familiari durante la guerra civile, c'è un interessante lavoro sul bergamasco [Quarenghi (ed.) 2003]. Per alcuni spunti su progetti di ricerca e di valorizzazione del patrimonio fotografico: Lusini (ed.) 1996. 


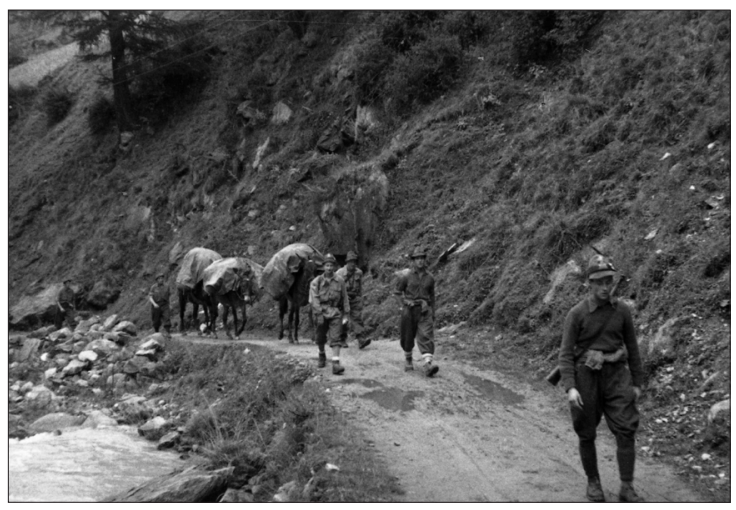

Fig. 1 Il viaggio di Ettore Serafino da Aosta alla val Pellice [Archivio famiglia Serafino] magini scattate tra il 1939 e il $1946^{12}$. I primi due raccolgono le foto da militare in Croazia, sul fronte greco-albanese, in Montenegro $^{13}$. Il terzo album comprende le fotografie realizzate nel periodo partigiano, e inizia con la documentazione del viaggio da Aosta a Bobbio Pellice del settembre del $1943^{14}$.

\section{L'estate del '44 in val Chisone}

Dopo queste brevissime note biografico-documentarie, occorre capire che cosa accadde in val Chisone nell'estate del 1944. Tra la fine di maggio e i primi di giugno $^{15}$ i tedeschi e i fascisti lasciarono l'alta valle, che per oltre due mesi fu dunque un territorio libero. È lo stesso Ettore Serafino a parlarne in uno scritto pubblicato poco dopo la liberazione [Serafino 1945, 10]:

Giugno 1944. La valle del Chisone, dal Villaretto al colle di Sestriere, viene ad

12 Oltre ai positivi di vario formato $(6 \times 9 \mathrm{~cm}, 9 \times 13,7.5 \times 10,10 \times 15,13 \times 18,5 \times 6)$, sono presenti tutti i negativi e gran parte dei provini. I tre album sono stati montati dallo stesso Ettore Serafino nel dopoguerra e contengono didascalie scritte in calce e sulle veline. Esiste un quarto album realizzato dal figlio Adolfo su richiesta del padre in anni più recenti contenente solo le fotografie partigiane, con nuove didascalie. Vista l'assenza di una schedatura della documentazione e di inventario dettagliato, i riferimenti archivistici presenti nel saggio saranno dunque sommari: la ricerca in corso prevede anche l'ordinamento del fondo fotografico. L'archivio fotografico è decifrabile solo attraverso un'indagine svolta sull'intero fondo personale, che comprende anche l'archivio cartaceo: circa 7 metri lineari di documenti e 38 faldoni. L'archivio cartaceo, parzialmente inventariato dal figlio Adolfo sulla base dell'ordinamento del padre, contiene documenti personali di Ettore Serafino: scritti giovanili, i suoi articoli per i giornali fascisti, poesie, l'epistolario, le carte delle bande partigiane comandate, ritagli di giornale, discorsi commemorativi pronunciati nel dopoguerra, ecc.

13 La ricerca in corso sul fondo prenderà in esame l'intera documentazione fotografica della guerra e cercherà di cogliere le logiche della sua composizione. Ad alcune domande occorrerà rispondere in modo preciso: perché sono così numerosi gli scatti relativi alla guerra nei Balcani (1321 positive)? Quali differenze rispetto alle fotografie del periodo partigiano (il terzo album comprende gli scatti tra l'8 settembre 1943 e il 1946: 662 positive di cui 262 riferite al periodo partigiano)? Quale uso veniva fatto delle immagini dal fronte? Come si procurava i rullini in guerra? Quale cultura fotografica aveva l'autore?

14 || racconto di quel viaggio e del suo 8 settembre rappresenta un nodo fondamentale nella memoria di Serafino, e tale aspetto si evince anche nel fondo fotografico: a esso sono dedicati 25 scatti.

15 Le varie versioni dei testimoni sono leggermente discordanti sul periodo: il comandante Marcellin ha scritto che i tedeschi e i fascisti lasciarono l'alta valle il 21 maggio [Marcellin 1966], altri parlano dei primi di giugno. Ci sono diversi lavori sulla Resistenza in valle, ai quali rimando [Trabucco 1984; Gay Rochat 1985; Boccalatte, D’Arrigo e Maida (eds.) 2009]. 
essere in mano dei patrioti di Marcellin. Se la sono conquistata si può dire paese per paese, catturando i presidi nemici o costringendoli a ritirarsi; i ponti saltati completano l'isolamento della valle. Sulla bella rotabile asfaltata di fondo valle sfrecciano le motociclette dei partigiani, i ragazzi finalmente scendono nei paesi, ne popolano le vie, i ritrovi. Ma fino a quando può durare indisturbata questa tranquilla vita di presidio?

Più o meno negli stessi mesi lo stesso accadde nelle valli limitrofe, ma la formalizzazione della zona libera ci fu solo in un caso, quello delle valli di Lanzo ${ }^{16}$. Nonostante ciò, il governo del territorio da parte delle formazioni partigiane fu esercitato ovunque: talvolta creando amministrazioni gestite dal Cln, talvolta insediando nuove giunte comunali, altre volte non modificando gli strumenti di governo ereditati dal fascismo.

Quel che accadde in val Chisone è ben testimoniato dalle parole di Rodolfo Sacco, classe 1923, partigiano della formazione autonoma dal maggio del 1944, dopo qualche mese trascorso col gruppo cattolico di Silvio Geuna:

Perosa era fermamente nelle mani della Repubblica [...]. Oltre Perosa cominciava una terra di nessuno. Non c'era nessuno a Meano, a Castel del Bosco assolutamente non c'era nessuno. Noi a Castel del Bosco andavamo sicurissimi di non essere molestati. Ho già detto che io ho sposato una valchisoniana, una di Castel del Bosco. Come l'ho conosciuta? Quando andavo in pattuglia mica chiacchieravo con la valchisoniana! Il nostro comandante era Eugenio Juvenal di Roure - adesso porta il nome Roreto - lui era di lì, aveva lì la sua casa, [...] e andava a Castel del Bosco a trovare una giovane nata nel ' 25 - lui era nato nel '23 - che di mestiere faceva il Conservatorio e l'Accademia Albertina [...]. Lui le faceva la corte, una corte accettata dalla famiglia, ma con le abitudini dell'epoca lui non poteva andare a trovare la morosa - non erano proprio fidanzati, lo stavano diventando - non poteva andare a trovarla da solo, ma doveva portarsi dietro qualcuno, e aveva trovato in me la persona giusta che andava, non faceva commenti, che non si impicciava. La Adriana Gay in questione aveva con sé, ospite sfollata con lei, una cuginetta a nome Amélie, ma tutti dicevano Lili, Lili Gay, i cui genitori erano in Marocco e la figlia in Italia per ragioni di studio, appoggiata allo zio... e io lì ho conosciuto questa connazionale del Marocco e poi la cosa è diventata importante [...]. Se con Juvenal andavamo a incontrare le ragazzine, vuol proprio dire che sapevamo che era tranquillo, che non creavamo pericolo alle persone che andavamo a trovare. Un partigiano che entrava in una casa metteva in pericolo quella casa se $i$ tedeschi sapevano che lui c'era entrato. Quindi era proprio terra di nessuno. Ma dove noi

16 Con l'eccezione della val di Susa, che non fu mai lasciata ai partigiani perché il suo controllo era considerato strategico: in primavera si formò un'amministrazione municipale a Prali in val Germanasca, zona che però aveva uno scarso interesse per il nemico; da fine giugno a fine settembre le valli di Lanzo furono governate dalle forze antifasciste; per circa un mese, da fine giugno ai primi d'agosto, pure la vicina val Pellice fu temporaneamente liberata dai nazi-fascisti. Per restare al Piemonte, oltre alle più note repubbliche partigiane, furono zone libere anche le valli Varaita e Maira da fine giugno a fine agosto e, per un mese, la Valsesia, tra giugno e luglio. 
avevamo pieno accesso, noi sì e gli altri no, era a partire da Castel del Bosco a venire in su. La vera terra di nessuno era Meano, che era già un pochettino sotto la nostra saltuaria presenza. Tutta Roure, tutta Mentoulle. [...] Dove invece eravamo proprio noi era Fenestrelle. Lì CO-MAN-DA-VA-MO noi! Imponevamo corvée ai civili, se capitava. Era una delle cose che capitava di fare, e i civili rispondevano con una dedizione totale ${ }^{17}$.

L'impressione di generale tranquillità si rileva anche nella memoria del comandante Marcellin, per esempio quando racconta dell'arrivo di Ada Gobetti verso fine giugno. Scrive Marcellin [Marcellin 1966, 106]:

volevo riceverla, ma prima volevo che qualcuno la riconoscesse, e ciò non fu facile. Poi finalmente trovai un partigiano torinese $[\ldots]$ il quale mi disse di conoscerla benissimo. Feci in modo che i carabinieri la fermassero e l'accompagnassero all'albergo Frezet, dove il mio uomo si recò e senza farsi notare poté vederla e riconoscerla.

Come in tutte le esperienze analoghe, il governo territoriale contemplò ovviamente l'impegno dei partigiani su più fronti, non solamente su quelli militari [Marcellin 1966, 107-108].

$\mathrm{Fu}$ anche necessario controllare un altro settore vitale della nostra organizzazione: l'ufficio affari civili. Convocato il responsabile, si provvide ad una specie di mobilitazione civile della zona occupata. Grazie al censimento già effettuato delle persone, dei quadrupedi, dei traini e di ogni altro mezzo utile, vennero inviate le lettere-precetto ${ }^{18}$.

Fu Sacco, studente in legge, allievo ufficiale che ispirava una certa fiducia al suo comandante Marcellin per «l'aria precocemente matura» ${ }^{19}$, a ricevere l'incarico, in realtà non ben precisato, di occuparsi degli affari civili insieme al segretario comunale di Fenestrelle, arruolatosi nella formazione col nome di battaglia Aurora: proprio Aurora sarebbe stato «la musa di un eventuale servizio organizzazione civile». Ma tutto era in fieri, tutto doveva essere inventato e sperimentato di volta in volta: l'amministrazione della zona liberata procedette dunque a tentoni. Come riconosce lo stesso Sacco, «non sapevamo che cosa dovevamo fare, io in quel momento non avevo ancora l'esperienza di aver governato su un vasto comune com'era allora Frossasco».

Nonostante la scarsa pianificazione, l'assenza di direttive dall'alto, la mancanza di competenze amministrative e le difficoltà dei partigiani di fronteggiare compiti

17 Intervista a Rodolfo Sacco del 27 marzo 2013.

18 I civili, per esempio, furono coinvolti nell'allestimento delle opere difensive.

19 Intervista a Rodolfo Sacco. 
nuovi e complessi in una valle accerchiata dai nemici, la formazione di Marcellin e di Serafino resse per diverse settimane la valle. Come? I partigiani esercitarono direttamente il potere coinvolgendo i civili, ma senza dar vita a Cln locali o ad altri organismi democratici, per l'autonomia che gli uomini di Marcellin rivendicarono sempre dai partiti politici.

Ed Ettore Serafino? Egli arrivò nella valle già liberata. Si aggregò infatti alla formazione autonoma nella seconda metà di giugno, dopo aver lasciato la val Pellice ed esser riuscito a fuggire dal carcere nel quale era stato rinchiuso a Pinerolo dalla Guardia nazionale repubblicana ${ }^{20}$. Al termine di un periodo delicato per il raggruppamento partigiano, durante il quale si aprì un conflitto tra alcuni comandanti, tanto che Marcellin minacciò le dimissioni, Serafino ottenne il comando del battaglione Monte Assietta. Il suo inquadramento si completò molto rapidamente, anche perché era una figura ben nota tra i partigiani autonomi: non solo infatti egli aveva incontrato Marcellin più volte precedentemente, ma conosceva personalmente molti alpini accorsi in valle per unirsi alle bande.

Giugno e luglio 1944 furono dunque un momento di relativa calma in val Chisone $^{21}$ : Serafino avrebbe potuto scattare qualche fotografia, lasciandoci la testimonianza visuale del clima che si respirava in quelle settimane estive. Ma non lo fece. Perché? Forse non si trovava nelle condizioni ottimali per dedicarsi alla fotografia: dov'era nel periodo della zona libera? Aveva a disposizione rullini? Ha scattato fotografie perse durante il conflitto oppure eliminate dall'archivio personale dopo la guerra?

\section{Presenze o assenze: il problema della datazione fotografica}

Prima di procedere con l'analisi, mi soffermerò sulla datazione delle fotografie del 1944. Bisogna infatti esser sicuri che il giovane alpino non avesse effettivamente lasciato alcuna immagine della zona libera' ${ }^{22}$.

20 II racconto della cattura e della fuga si trova in alcune lettere: Archivio famiglia Serafino (d'ora in poi AS), Corrispondenza, lettere del 27 giugno 1944 e del 30 giugno 1944.

21 II comando della formazione era molto preoccupato dai vari attacchi delle truppe nazi-fasciste provenienti sia dal fondo valle sia dalla confinante val Susa.

22 A seguito di un sondaggio preliminare effettuato all'inizio della ricerca, mi ero convinto che una parte delle immagini fosse effettivamente riconducibile al periodo della zona libera, ma ovviamente si trattava di ipotesi da verificare sul campo. Per la storia dell'archivio familiare e per le sue caratteristiche, non mi sono trovato dinnanzi a documenti inventariati e schedati con precisione, dunque ho dovuto dedicarmi a questa operazione in tutta la prima fase del lavoro, cercando continuamente di incrociare la storia del movimento partigiano in val Chisone, le vicende biografiche di Ettore Serafino e le sue fotografie. In questa fase del lavoro l'apporto di Adolfo Serafino è stato determinante. 
Per decifrare l'archivio fotografico di Ettore Serafino ho integrato informazioni di varia natura e provenienza [Krauss 1999]: le didascalie (ove presenti) scritte dallo stesso autore nelle pagine degli album; le notizie raccolte una decina di anni fa dai figli sui singoli scatti; i documenti del fondo cartaceo dell'archivio privato, anzitutto la corrispondenza; numerosi diari, memorie e interviste di partigiani della stessa formazione; vari fondi archivistici sul movimento partigiano in val Chisone. A ciò si è aggiunto lo studio dell'oggetto fotografia [Giovannini Luca 2014]. Gli album sono stati ordinati dallo stesso Serafino e riproducono la medesima sequenza degli scatti rilevabile dai negativi, fortunatamente conservati. Da una serie di verifiche ${ }^{23}$, l'ordinamento è risultato fedele e cronologicamente corretto. Appurato ciò e individuati i rullini contenenti le fotografie potenzialmente riconducibili all'estate del '44, poiché comprese tra due estremi cronologici certi, cioè la primavera del '44 e l'inizio dell'inverno successivo, è stato necessario procedere a una datazione meno vaga ${ }^{24}$. L'attenzione rivolta alle persone fotografate ha permesso di affinare la periodizzazione $a$ quem $^{25}$ : alcuni scatti ritraggono infatti Eugenio Juvenal (immagine 3) e Adolfo Serafino, il fratello di Ettore, uccisi a Cantalupa il 4 novembre 1944 ${ }^{26}$ (immagine 7), e altri ritraggono il partigiano Gianni Gay con il lutto al braccio per la morte del fratello Enrico, avvenuta il 19 agosto 1944 in uno scontro con i nemici (immagine 6).

Fatta questa selezione, restavano ancora da vagliare una quindicina di fotografie: dalla mera osservazione dei vestiti, delle pose, delle espressioni, delle situazioni e dei paesaggi, esse si sarebbero potute attribuire all'estate del '44, ma le didascalie dell'autore le facevano risalire al settembre e all'autunno di quell'anno. A che cosa credere, dunque? Agli elementi che si rilevano dall'osservazione della fotografia o alla testimonianza di Ettore Serafino? Il dubbio mi pareva ragionevole, non solo perché capita spesso che i testimoni confondano i loro ricordi, ma anche

23 Mi riferisco alla consultazione della corrispondenza presente nell'archivio familiare di Serafino, all'incrocio tra le foto stampate e i negativi e alla testimonianza del figlio Adolfo, che ha dedicato molta attenzione alla conservazione dell'archivio familiare, in particolare di quello fotografico.

24 Anche per scartare l'ipotesi che l'autore avesse commesso errori nella stesura delle didascalie o per tentare di completare le sue informazioni, talvolta troppo generiche.

25 L'identità delle persone è rintracciabile grazie alle didascalie presenti e a quelle scritte successivamente dal figlio Adolfo in collaborazione con il padre.

26 Dalla corrispondenza di Ettore con la famiglia dopo l'uccisione di Adolfo, risulta che le ultime immagini del fratello siano del mese di ottobre. 
perché gli avvisi a diffidare di alcune didascalie mi provenivano da più direzioni ${ }^{27}$. Dovevo però uscire da questo vicolo cieco. Come?

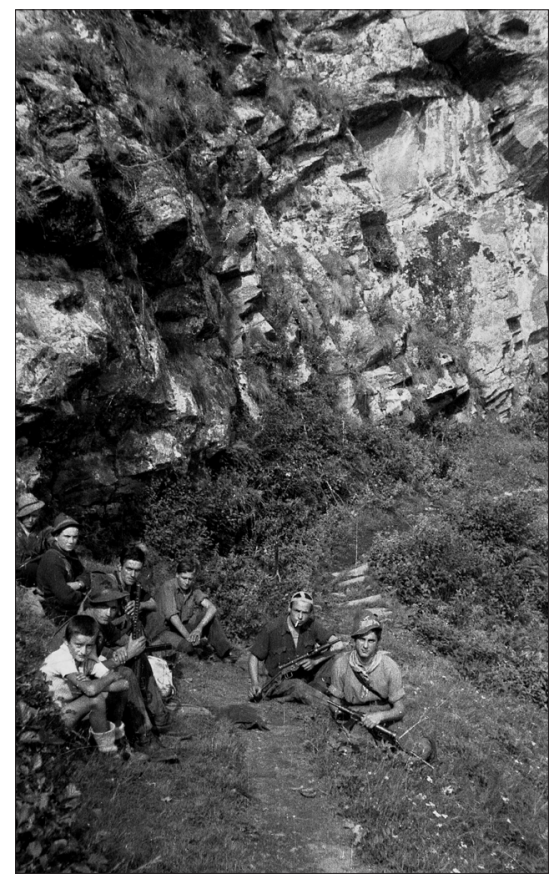

Fig. 2 Settembre 1944, partigiani in val Chisone [Archivio famiglia Serafino]

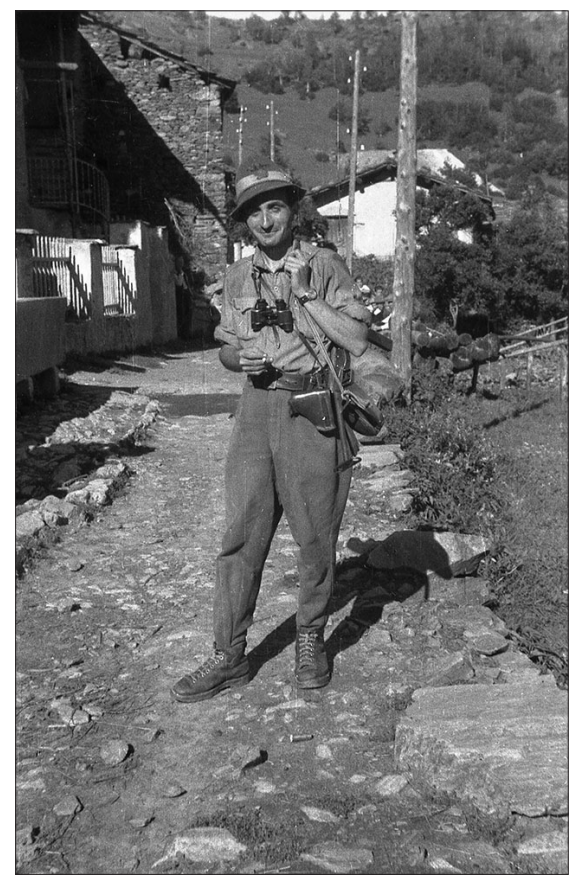

Fig. 4 Autunno 1944, Borgata Fontane (val Germanasca). Ettore Serafino [Archivio famiglia Serafino]

Fig. 3 Autunno 1944, Borgata Fontane (val Germanasca). Il partigiano a destra è Eugenio Juvenal [Archivio famiglia Serafino]

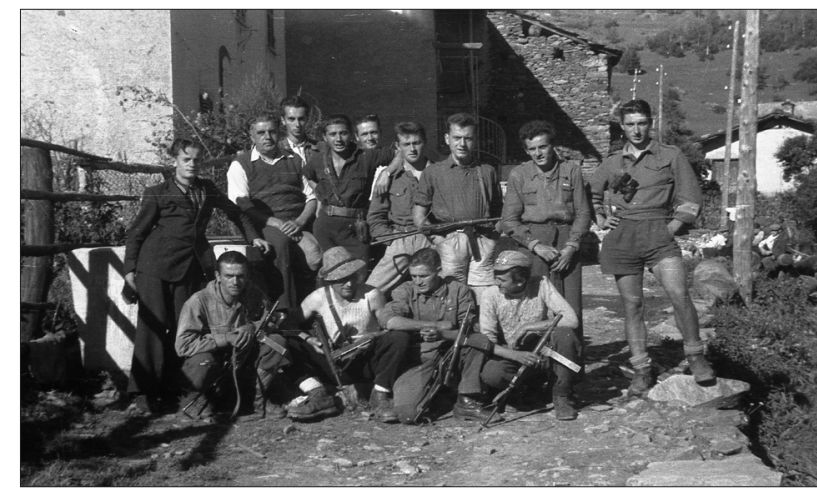

27 Alcune informazioni sulle condizioni meteorologiche in valle desumibili dalle immagini non ci aiutano a uscire da questa confusione: alcune testimonianza parlano di una prima nevicata sul Col Clapis (oltre $2.700 \mathrm{~m}$ ) a inizio settembre, mentre in una fotografia datata da Serafino "autunno '44" appare il vicino Bric Ghinivert (3037 m) spoglio, e i partigiani immortalati in quell'immagine e in quelle seguenti appaiono vestiti con abiti leggeri. Inoltre, il figlio Adolfo e l'amico di famiglia Gianvittorio Avondo, storico della Resistenza del pinerolese che ben conosceva Ettore Serafino, avevano i miei stessi dubbi. 


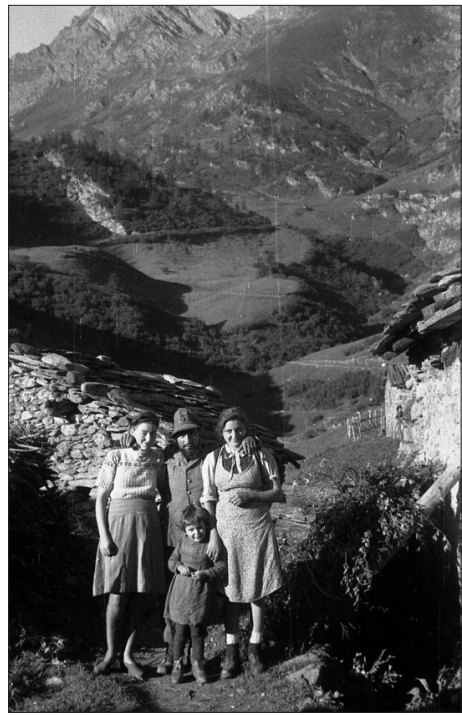

Fig. 5 Autunno 1944, Balme di Rodoretto (val Germanasca). Enrico Poet con moglie, sorella e figlia [Archivio famiglia Serafino]

Fig. 7 Autunno 1944, Inverso di Pinasca. Adolfo (sinistra) ed Ettore Serafino (destra) [Archivio famiglia Serafino]

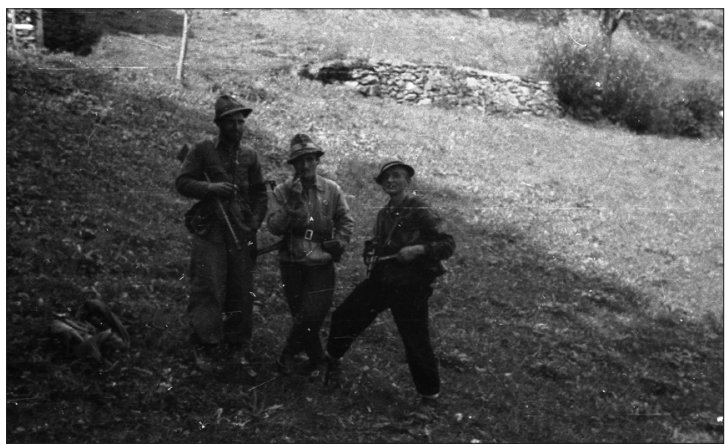

Fig. 6 Autunno 1944, Inverso di Pinasca. A sinistra Gianni Gay con il lutto a braccio, al centro Ettore Serafino [Archivio famiglia Serafino]

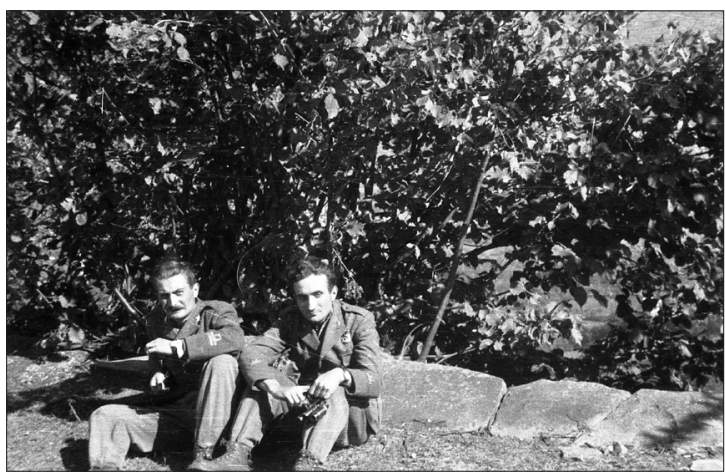

Ho cominciato a cercare informazioni in altre direzioni esaminando diverse fonti: memorie, diari, testimonianze, documentazione ufficiale della formazione, carte personali. Ho trovato le indicazioni più confortanti all'interno della corrispondenza di Ettore, conservata nell'archivio cartaceo della famiglia. Grazie alle lettere, infatti, si possono ricostruire molti movimenti di Serafino in quel periodo. Dove si trovava Ettore ai primi di settembre del 1944? Nella limitrofa val Germanasca, da dove partirono diversi messaggi indirizzati alla famiglia ${ }^{28}$. I luoghi citati nella corrispondenza corrispondono con quelli delle didascalie ${ }^{29}$. L'incrocio delle immagini, dei nomi delle persone immortalate, degli spostamenti di Ettore sembra dimostrare che il giovane comandante partigiano non fece ricorso alla sua Kodak fino alla fine di agosto o fino ai primi di settembre del 1944; egli tralasciò dunque

28 Si veda per esempio AS, Corrispondenza, lettera del 3 settembre 1944. Della val Germanasca era originario Enrico Poet, uno dei partigiani immortalati in queste sequenze.

29 Una lettera è scritta da Campo di Salza il 3 settembre, un'altra da Balme di Rodoretto il 4. 
di imprimere sulle sue pellicole le immagini del periodo della zona libera ${ }^{30}$.

\section{La zona libera senza immagini}

Appurata la datazione delle immagini del 1944, iniziamo la trattazione dei vari fattori che hanno influito sulla memoria fotografica della zona libera in val Chisone. Prenderò anzitutto in esame le variabili locali, imputabili al fotografo o alle vicende partigiane della valle, e passerò in un secondo momento ad analizzare quelle sovralocali.

La prima da considerare è tanto banale quanto essenziale, cioè la disponibilità di rullini. Rispetto agli scatti realizzati precedentemente, quelli riferiti certamente all'estate-autunno del 1944 (e molto probabilmente ai mesi di settembre-ottobre) sono impressi in un nuovo rullino di marca differente, e ciò potrebbe indicare il cambiamento del fornitore ${ }^{31}$. Quali informazioni abbiamo sulle modalità usate da Serafino per procurarsi le pellicole? Per il periodo partigiano sappiamo che Ettore si rivolgeva abitualmente a un fotografo di Pinerolo con il negozio vicino alla casa di famiglia, al quale chiedeva anche lo sviluppo ${ }^{32}$. Non siamo però in grado di dire quando Serafino cominciò a servirsi da questo professionista. Prima dell'estate? Dopo? Ma se anche non avesse avuto sottomano qualche rullino, il periodo di relativa tranquillità in valle non lo avrebbe aiutato a trovarne di nuovi? Al momento non mi è facile rispondere a tali quesiti, se non per via indiziaria. Ettore Serafino si muoveva parecchio nell'alta e nella bassa valle, oltre che nelle vallate vicine (Susa, Germanasca, Pellice) ${ }^{33}$, e aveva tanti rapporti con amici e conoscenti della zona, partigiani e civili: è dunque molto improbabile che si fosse rassegnato di fronte alle difficoltà di reperimento dei rullini. La sua storia di

30 Visto che si tratta certamente di fotografie della formazione autonoma della val Chisone, non si possono avere dubbi sulla datazione a quo: Serafino, come detto, si unì al gruppo di Marcellin alla fine di giugno del 1944. E inoltre improbabile che gli scatti riguardino il mese di agosto, poiché i partigiani furono impegnati a fronteggiare l'imponente offensiva tedesca, poi ripararono in Francia e in quelle settimane Serafino svolse un'estenuante operazione di collegamento e di riorganizzazione dei vari gruppi dispersi dall'operazione militare nazi-fascista.

31 Non è però facile dare rilievo a questo aspetto, anche perché Serafino cambiò diverse marche durante la guerra: prima l'Agfa, poi la Gevaert Belgium (è questo il caso dei rullini relativi all'estate-autunno del 1944), poi di nuovo un Agfa, infine un Kodak e ancora un Agfa. Sapere da chi si procurasse i rullini Serafino ci aiuterebbe a interpretare le sue fotografie: potremmo capire se l'assenza di foto del periodo della zona libera sia dipesa da mere ragioni materiali.

32 Mi ha dato questa informazione Gianvittorio Avondo. Al momento non sono riuscito a reperire altre notizie su questo fotografo e sono alla ricerca (non facile) di informazioni sulla disponibilità di materiale fotografico in zona durante la guerra.

33 Dalla corrispondenza risulta per esempio diverse volte a Pinerolo nel mese di giugno (oltre che nei mesi precedenti), quando fu poi arrestato. 
fotografo della Resistenza dimostrerebbe proprio il contrario, poiché, nonostante le avversità, egli trovò sempre il modo di portare avanti la sua passione ${ }^{34}$. Escludo dunque che l'assenza di scatti del periodo della zona libera sia dipesa dall'indisponibilità della pellicola da caricare nella macchina fotografica.

Ma sulla consistenza del fondo fotografico di Ettore Serafino possono aver influito altre vicende prettamente personali. Prendiamone in considerazione ancora una: Serafino si unì alla formazione a fine giugno. Perché allora non scattare nemmeno una foto in tutto il mese di luglio? Si potrebbe sostenere che il neo arrivato avesse bisogno di un periodo di ambientamento ${ }^{35}$. Ma Serafino, che divenne rapidamente uno stimato comandante, non ebbe in realtà grossi problemi di integrazione nella nuova realtà, anche perché conosceva da tempo molti partigiani della formazione autonoma, in gran parte ex alpini, e assunse da un giorno all'altro il comando di un battaglione. La condizione di nuovo arrivato non può spiegare l'assenza di scatti: al contrario, la vita tranquilla di quel mese e la sua familiarità con i compagni avrebbero potuto incentivarlo a moltiplicare le istantanee. D'altro canto, l'ingrossamento della formazione nell'estate avrebbe potuto facilmente determinare l'incremento delle fotografie, magari proprio per il bisogno di documentare questa nuova fase della guerra partigiana, che, nonostante le difficoltà logistiche, portava nuovi combattenti in montagna, oppure per rispondere alla richiesta degli ultimi arrivati di poter disporre di un proprio ritratto. Andrebbe maggiormente studiata l'origine delle fotografie partigiane: quali pressioni ricevevano i fotografi da parte degli altri combattenti? Quanto era alta la domanda di fotografie nelle bande? A giudicare dalle tipologie di scatti più diffuse, essa doveva essere piut-

\footnotetext{
34 Nelle settimane della zona libera la valle era isolata da posti di blocco delle truppe nazi-fasciste, e ciò in teoria avrebbe potuto ostacolare il rifornimento di rullini. Tuttavia dalla testimonianza del figlio Adolfo e da alcune lettere (per esempio, AS, Corrispondenza, lettere di Giovanni Turvani e di Nini Coucourde) si evince che Ettore si appoggiasse ad alcuni amici per far consegnare o ritirare i rullini sviluppati: teneva molto alla sua passione, dunque organizzò le stampe usando la sua rete sociale. Ė dunque probabile che usasse le stesse relazioni per procurarsi le pellicole.
}

\footnotetext{
35 Del resto le fotografie della Resistenza si fondano su un criterio di base: la fiducia tra il fotografo e il fotografato. Questa annotazione permette di richiamare alcune questioni metodologiche relative allo studio delle immagini. Difficilmente il lavoro dello storico sulle fotografie potrebbe limitarsi agli elementi estetici, per lo più fuorvianti o comunque parziali. La produzione fotografica va infatti anzitutto contestualizzata seguendo le indicazioni suggerite dagli storici sociali dell'arte, che hanno avuto il grande merito di mettere in discussione i tradizionali approcci disciplinari allargando l'osservazione a elementi esterni all'opera d'arte, e offrendo così agli storici dell'arte nuovi ambiti di ricerca e nuove domande. Tale impostazione si è sviluppata a partire da un presupposto valido anche nell'esame della fotografia: il significato delle immagini è desumibile solo se lo studioso è in grado di ricostruire la trama che lega tra loro fattori diversi, come il ruolo dell'autore, le caratteristiche (estetiche e materiali) dell'opera, la tipologia di pubblico a cui è destinata, l'influenza della committenza, le forme e gli esiti della ricezione. Per interpretare le opere, spesso anche per datarle, occorre dunque estendere l'analisi al contesto di produzione e a quello di ricezione: tutti insieme identificano lo spazio entro il quale un determinato oggetto acquista rilevanza simbolica [Castelnuovo e Ginzburg 1979; Castelnuovo 1985; Baxandall 2000; Ginzburg 2004; Giovannini e Pierobon (eds.) 2014]
} 
tosto elevata ${ }^{36}$. Tali quesiti si legano a un problema più generale, di cui sappiamo ancora troppo poco: la circolazione delle immagini in guerra.

\section{Memoria individuale e memoria locale}

Le traiettorie individuali non spiegano da sole i tratti salienti delle fotografie partigiane. I partigiani-fotografi erano immersi in contesti sociali e culturali che ne modificarono e influenzarono lo sguardo ${ }^{37}$. Quali erano e come investigarli? Torniamo al nostro case study e chiediamoci: esistono immagini del 1944 in val Chisone oltre a quelle di Serafino? Qualcun altro ha documentato la zona libera? La risposta è negativa. Non è che siano mancati del tutto i fotografi della Resistenza in valle, ma nelle fonti al momento disponibili non si riscontrano elementi tanto difformi da quelli finora emersi dall'analisi dell'archivio di Ettore Serafino $0^{38}$ o non vi sono scatti della zona libera, oppure le immagini non sono distinguibili dal topos classico della produzione partigiana. Alcune variabili locali, esterne anche se non estranee agli individui, agirono dunque sulla costruzione della memoria fotografica della zona libera in val Chisone. Dovremmo cercare di capire quali. Mi sono così immerso nell'analisi di diari, memorie, articoli e interviste di partigiani della formazione autonoma val Chisone. Sono infatti numerosi gli scritti e le testimonianze degli ex combattenti, già del dopoguerra. I riferimenti ai mesi della zona libera sono sistematicamente limitati all'organizzazione militare e alla difesa della valle. Pochi cenni si trovano per esempio nel diario del comandante Maggiorino Marcellin [Marcellin 1966], nessun racconto nel libro di memorie scritto da Ettore Serafino nel 2001 [Serafino 1994; Serafino 2001], così come nei diari partigiani di Franco Obert [Obert 1993] e di Gino Rostan [Rostan 2003]. Poco, pochissimo anche altrove, sia in un opuscolo preparato all'indomani della liberazione da diversi partigiani per raccontare la Resistenza in valle [Noi Alpini della Val Chisone 1945], sia nei diversi diari conservati nel fondo dei fratelli Va-

36 Tale problema rimanda a un tema che cercherò di indagare nella ricerca sul fondo Serafino: qual era la cultura fotografica dei giovani partigiani, fotografi amatoriali e non? L'utilizzo delle fotografie in guerra si lega infatti al generale uso della fotografia tra i ceti popolari in quel periodo. Secondo l'approccio dei visual studies, l'immagine non può essere considerata separatamente dalla cultura visuale che la esprime [Alpers 1984; Evans e Hall (eds.) 1999].

37 Rimando proprio all'approccio tipico dei visual studies.

38 Sulla val Chisone e sulla formazione autonoma di Marcellin si trovano diverse immagini classiche di gruppi in posa, per lo più realizzate dopo la liberazione. Per esempio, ffr. Archivio privato famiglia Obert. Ringrazio Gianvittorio Avondo, esperto della Resistenza nel pinerolese, per l'aiuto fornitomi nella ricerca di altre fotografie della Resistenza in valle. 
nossi, alcuni redatti poco dopo la fine della guerra ${ }^{39}$.

La memoria ufficiale della formazione e della Resistenza in valle ha dunque lasciato poco spazio all'esperienza della zona libera. Questo silenzio, questa omissione, riflette quanto riscontrato nell'esame della documentazione fotografica. Eppure, come abbiamo visto, quell'esperimento coinvolse partigiani e civili in nuove forme di governo territoriale e a queste fanno cenno alcuni testimoni, $\mathrm{i}$ quali però non approfondiscono mai l'argomento. La memoria partigiana degli autonomi si è soprattutto concentrata sulle vicende militari e sui caduti, e quella dell'estate del 1944 è quasi integralmente egemonizzata dai racconti dell'operazione Nachtigall (che segnò la fine della zona libera), della reazione partigiana, dell'abbandono della valle in agosto, del ripiegamento in Francia, del ritorno in Italia e della riorganizzazione della formazione verso la fine della stagione. Questo resoconto, spesso molto dettagliato, ha offuscato il periodo precedente e lo ha ridotto a parentesi. Ma la marginalizzazione della zona libera nella memoria locale si spiega anche con il carattere prettamente militare della brigata: i membri della formazione autonoma hanno sempre rivendicato, tanto in guerra quanto dopo, la piena autonomia dai partiti del Cln. Lo spiega bene Rodolfo Sacco: «noi vedevamo nel Cln l'autorità costituita $[\ldots]$ ma sapevamo che i partiti politici cercavano di mettere la loro uniforme» ${ }^{40}$. Se la storia del territorio liberato della valle rientra in quella che Roberto Battaglia definì la prima fase delle esperienze di autogoverno partigiano, in cui tutto doveva essere inventato e sperimentato con la netta prevalenza degli aspetti amministrativi su quelli politici, ciò che distinse la val Chisone fu la modalità di gestione del territorio operata dalla formazione autonoma: la tipologia di amministrazione esercitata in valle, cioè un governo militare che si avvaleva dell'opera dei civili, condizionò profondamente la memoria di quelle settimane di libertà. Il modello di governo del territorio fu infatti la mera estensione dell'organizzazione del movimento partigiano di Marcellin e Serafino, e così viene ricordato ${ }^{41}$. Nel tentativo di decifrare la cultura fotografica dei partigiani in valle, un elemento da considerare riguarda i valori prevalenti tra gli autonomi: la centralità della dimensione militare, che caratterizzò la vita partigiana in val Chisone prima, durante e dopo la zona libera, si è evidentemente

\footnotetext{
39 Archivio Istoreto, fondo Vanossi, b. 1.

40 Intervista a Rodolfo Sacco. Su questo punto rimando alla già citata intervista a Ettore Serafino e nuovamente al diario di Marcellin.

41 Proprio la presenza della formazione autonoma limitò o impedì I'affermarsi di esperienze democratiche diffuse sul territorio, come accadde in altri contesti.
} 
riverberata anche sulla memoria fotografica dell'estate del $1944^{42}$. In altre parole, in quel contesto partigiano si formò una specifica cultura resistenziale che ha condizionato la rappresentazione e il ricordo di certi eventi, e tale aspetto è rilevante nell'interpretazione delle fotografie.

\section{La funzione della fotografia della Resistenza e le rappresen- tazioni delle zone libere}

Le culture visuali sono il frutto di complesse interdipendenze tra molteplici fattori che non è sempre facile portare alla luce. Nel caso preso in esame il carattere prettamente militare della formazione ha giocato un ruolo fondamentale nel forgiare una precisa memoria fotografica, ma non possiamo ignorare l'esistenza di altre cause non strettamente collegate al contesto locale e di banda. A condizionare le forme rappresentative dell'estate del 1944 possono infatti aver agito variabili esogene e non riconducibili esclusivamente alla Resistenza in valle, e per capirlo ho esteso l'esame ad altri fondi fotografici. Nell'analisi - pur parziale - delle fotografie disponibili ho cercato eventuali tratti peculiari che distinguessero in modo significativo le immagini realizzate nelle varie zone libere dai caratteri prevalenti della fotografia resistenziale; ciò permetterebbe infatti di identificare una vera e propria memoria fotografica dell'esperienza e un preciso genere fotografico: la fotografia delle zone libere. Dovevo anzitutto farmi un'idea della produzione realizzata nei vari contesti, per poi setacciare il materiale alla ricerca di raffigurazioni tipiche del periodo, per esempio scene dell'amministrazione partigiana, del rapporto con i civili, del lavoro di partigiani e civili, delle opere di difesa, delle elezioni, delle riunioni, della distribuzione di alimenti, della giustizia, in altre parole di qualunque segno in grado di testimoniare l'atmosfera speciale che si respirò per poche settimane nelle varie comunità liberate.

Ho così visionato la documentazione relativa a molti altri casi analoghi in diversi fondi archivistici e in numerose pubblicazioni, procedendo per cerchi concentrici: dalla provincia di Torino al Piemonte e alle principali esperienze dell'Italia

42 Senza allontanarci troppo e senza richiamare le esperienze più note delle repubbliche partigiane, nelle vicine valli Germanasca e Lanzo, in cui operavano formazioni garibaldine e di Giustizia e Libertà, ci furono esperienze di partecipazione democratica, si formarono Cln locali, si crearono giunte municipali nuove. 
centro-settentrionale ${ }^{43}$. Ho rivolto l'attenzione ad aree come le valli di Lanzo, dove si formalizzò, nell'estate del 1944, l'esistenza della zona libera, e, sempre in Piemonte, alla valle Maira e alle più note repubbliche partigiane dell'Alto Monferrato, delle Langhe e dell'Ossola.

Quale l'esito di questo ampio spoglio documentario? Ogni situazione andrebbe analizzata a sé poiché il racconto fotografico della Resistenza dipende largamente dalle caratteristiche del movimento partigiano nelle diverse località, dunque per ogni caso sarebbe utile procedere ad approfondimenti mirati come quello condotto per la val Chisone. Per gli obiettivi del presente lavoro, in questa sede mi limito a restituire uno sguardo d'insieme e a presentare gli elementi comuni e prevalenti delle raccolte. In base alle attuali disponibilità documentarie, emergono tre particolarità. In primo luogo, nella maggior parte dei casi le fotografie delle zone libere risultano scarse o del tutto assenti ed è inconsueto imbattersi in mirate campagne fotografiche condotte nelle settimane di libertà; l'esempio di Montefiorino sembra in tal senso un unicum $^{44}$. In secondo luogo, sono estremamente rare le immagini raffiguranti situazioni, eventi o attività chiaramente riconducibili al racconto che di quei momenti hanno fatto i testimoni. Infine, risalta una tipologia di fotografia resistenziale in nulla differente da quella realizzata nei mesi precedenti o in quelli seguenti: spiccano infatti i ritratti di individui o di gruppi di partigiani in posa, qualche volta con alcuni civili, spesso parenti dei combattenti più o meno coinvolti nella guerra di liberazione ${ }^{45}$.

Lo studio della documentazione fotografica di un campione più ampio di esperien-

43 Rimando alle immagini conservate in vari fondi, tra cui: Archivio Istoreto, fondo Mautino; ivi, Fondazione solidarietà nazionale, in particolare le fotografie di vita partigiana in VP 2/203; ivi, fondo Resistenza Langhe; Archivio fotografico Centro di documentazione di storia contemporanea e della resistenza nelle Valli di Lanzo Nicola Grosa; Archivio fotografico del Comitato Resistenza Colle del Lys; Archivio fotografico di Corti Olimpio e Aldo. Ho visionato molte immagini pubblicate in numerosi volumi [Bravo 1965; Oliva 1983; Frassati (ed.) 1984; Remaggi, Silingardi e Teodoro 1994; Vottero Fin 1994; Battaglia e Silingardi (eds.) 2004; Buvoli e Nigris 2004; Del Boca (ed.) 2004; Silingardi 2005; La stampa ed i mezzi di comunicazione dei partigiani e della Repubblica dell'Ossola 2006; Lenzotti 2009; La Repubblica prima della Repubblica 2011; Buvoli 2013; Vallauri (ed.) 2013]. Lo stesso si riscontra nelle immagini di ben note esperienze democratiche realizzate fuori dai confini piemontesi, come a Montefiorino e nella Carnia.

44 È noto, per esempio, che a Montefiorino fu chiesto espressamente al fotografo-barbiere Aldo Corti di realizzare scatti di gruppi di partigiani, e ciò risulta molto bene dall'esame del suo fondo fotografico. Le fotografie contenute nell'Archivio fotografico di Corti Olimpio e Aldo sono visionabili sul sito http://raccoltafoto.comune.modena.it/index.html (ultima consultazione 27 maggio 2015).

45 Esiste qualche rara immagine che non ritrae gruppi di partigiani in posa durante il periodo delle zone libere. Per esempio, nelle valli di Lanzo qualche scatto testimonia la ricostruzione di un ponte a opera dei partigiani, o nell'Ossola una manciata di foto ritraggono il trasferimento in Svizzera della Giunta di governo quando iniziò la controffensiva nazi-fascista, nell'ottobre. Tuttavia tali eccezioni, che andrebbero comunque approfondite (se nel caso di Lanzo è possibile che l'autore fosse un membro della formazione, in quello dell'Ossola esse furono realizzate da un ufficiale svizzero della frontiera), non modificano le caratteristiche generali della fotografia del periodo. 


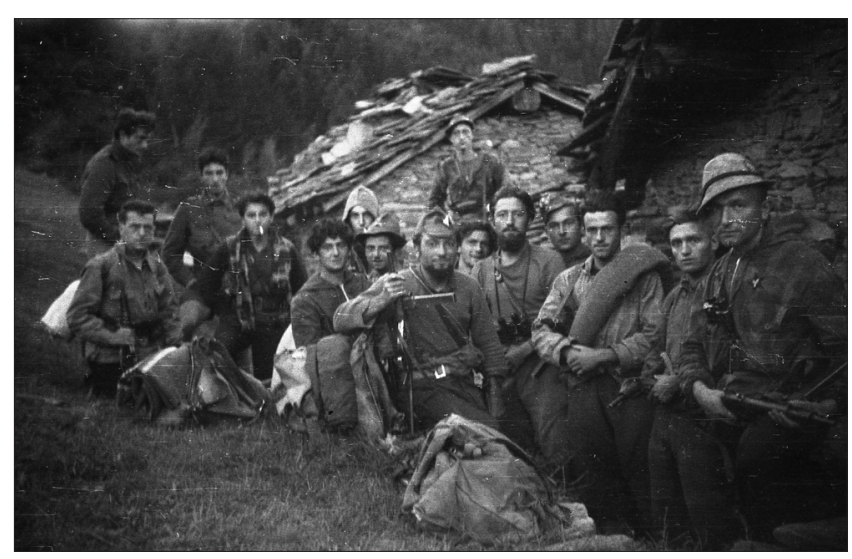

Fig. 8 Settembre 1944, partigiani in val Chisone [Archivio famiglia Serafino] ze simili a quella della val Chisone, talvolta pure politicamente più mature, introduce nell'interpretazione della rappresentazione fotografica delle zone libere fattori estranei alle vicende biografiche dei singoli fotografi ed esterni ai contesti locali. Gli obiettivi che i vari fotografi della Resistenza rivolsero in diverse aree dell'Italia occupata verso le comunità liberate non ebbero evidentemente lo scopo eminente di documen ${ }^{4 t}$ tare 10 "spirito del tempo", cioè il parziale e temporaneo ritorno delle libertà e la sperimentazione di forme di autogoverno che sancissero la discontinuità con il fascismo. Essi si concentrarono sui ritratti perché lo sguardo fotografico veniva conti-

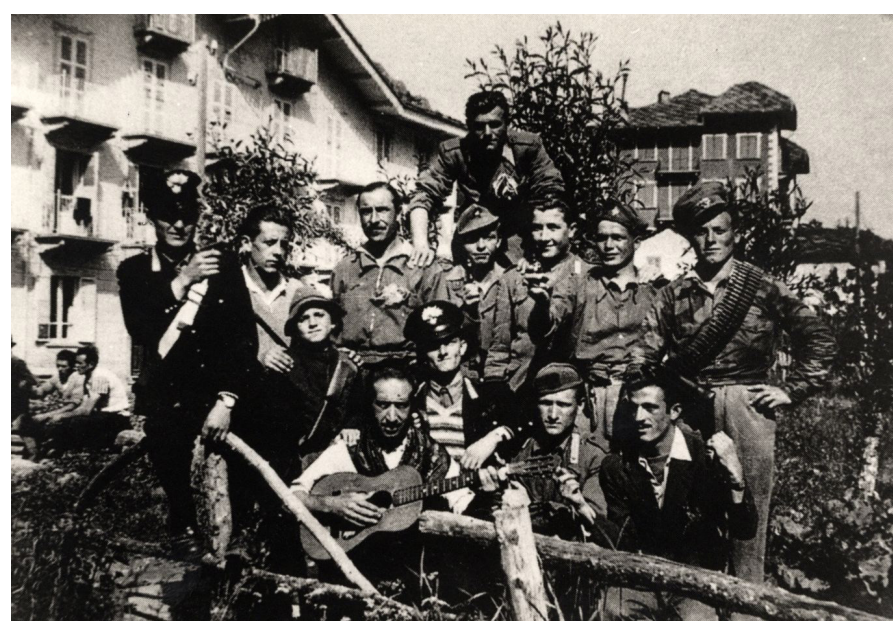

Fig. 9 Estate 1944, gruppo partigiano a Usseglio, nelle valli di Lanzo [Centro di documentazione di storia contemporanea e della resistenza nelle Valli di Lanzo Nicola Grosa] nuamente attirato da altro, anzitutto dal bisogno dei combattenti di lasciar traccia della propria azione e di comunicare a casa la propria esistenza. Questa tipologia particolare e molto diffusa di fotografia partigiana ebbe dunque il sopravvento, ed essa aveva

46 Al momento della liberazione si verificò un impressionante incremento di questo tipo di scatti, con un significato che, a guerra finita, assunse però contorni diversi. 


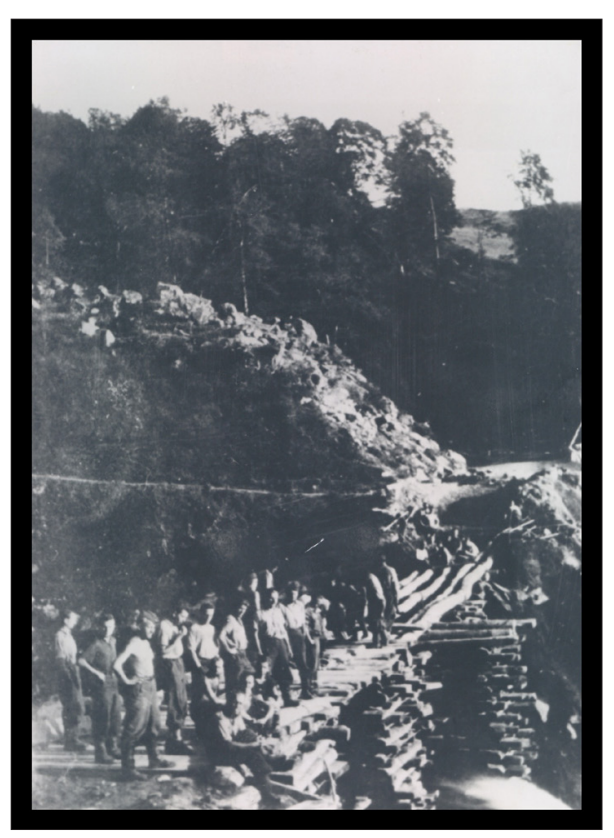

Fig. 10 Estate 1944, ricostruzione di un ponte a opera dei partigiani nelle valli di Lanzo [Centro di documentazione di storia contemporanea e della resistenza nelle Valli di Lanzo Nicola Grosa]

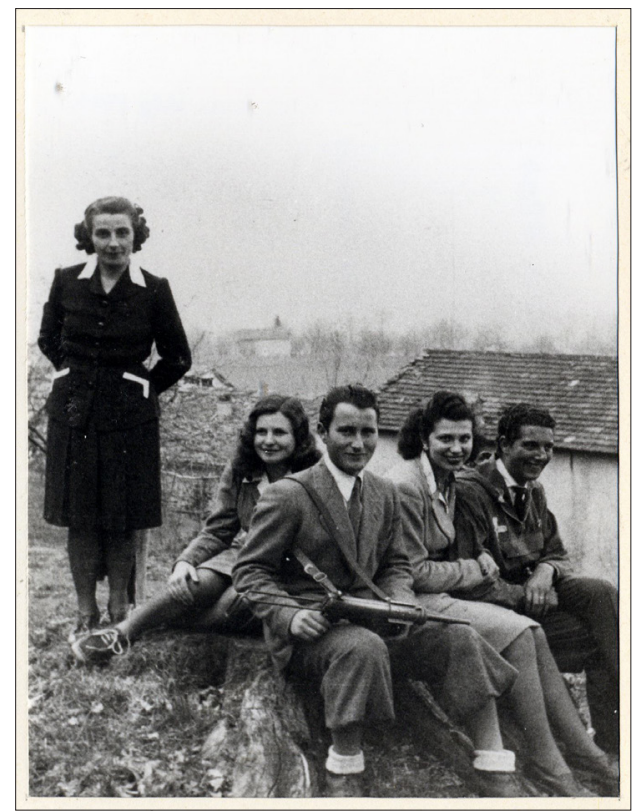

Fig. 11 Gruppo di partigiani in Valchiusella nell'inverno 1944-1945 [Archivio Istoreto, fondo Novascone]

una elementare funzione di testimonianza, ancora poco studiata, che prescindeva dal clima instauratosi nelle zone libere ${ }^{47}$. In banda, infatti, non mancarono mai i momenti di relativa calma e tranquillità, quelli in cui potersi dedicare alle chiacchiere, alle letture, al riposo e, quando si presentava l'occasione, a farsi fotografare, dunque gli album della Resistenza sono pieni di ritratti di gruppi in posa.

Al pari della storia sociale dell'arte, che sottolinea l'interdipendenza tra autori, pubblico e committenza, anche nello studio delle fotografie della Resistenza il contesto di produzione dell'immagine si lega quindi a quello di circolazione, cioè di ricezione [Gombrich 1965; Gilardi 1976; Bourdieu (ed.) 1972; Castelnuovo 1985; Haskell 1989; Freedberg 1993] $]^{48}$. In tempo di guerra, l'occhio del fotografo incrociò infatti le richieste dei soggetti fotografati, spesso committenti sui generis. Da questa interazione deriva la monotonia delle fotografie di gruppo

47 Nonostante il rischio che ciò comportava, circolarono tantissime immagini. Il problema dell'uso della fotografia della Resistenza in guerra come testimonianza mi sembra ancora in gran parte da esplorare. Per farlo, occorrerebbe trovare la giusta documentazione per studiare le fasi di sviluppo dei rullini e la circolazione delle immagini, cioè la loro prima ricezione.

48 Un utile riferimento nell'esame dei processi comunicativi è la teoria della pertinenza di Dan Sperber, Deirdre Wilson [Sperber e Wilson 1993]. 


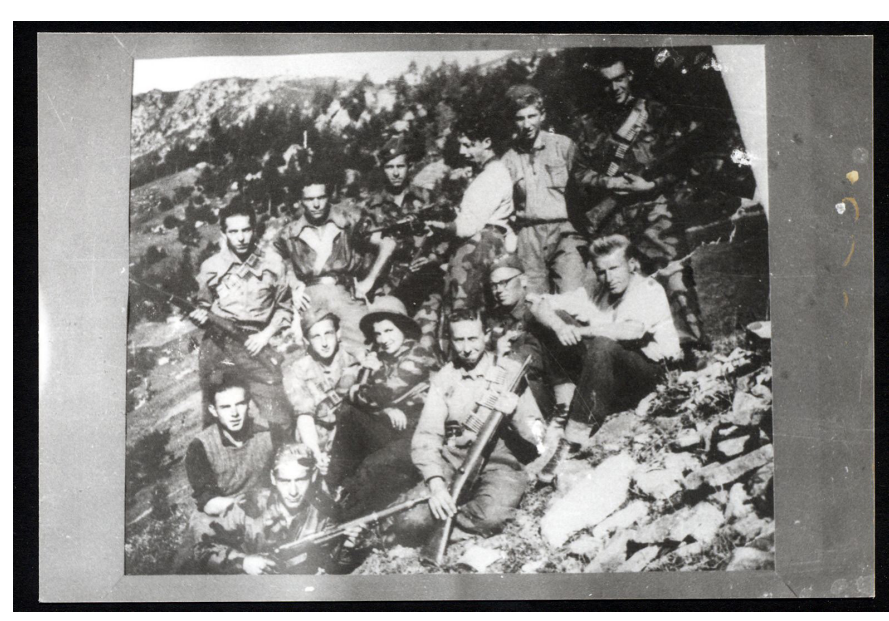

Fig. 12 Gruppo di partigiani della $41^{a}$ Brigata Garibaldi [Archivio Istoreto, fondo Dal Mas] dei resistenti, in cui compaiono soprattutto giovani maschi in posa che fanno spesso sfoggio di fucili, pistole e mitragliatori ${ }^{49}$ e che accentuano gli elementi militari della guerra partigiana. Questa autorappresentazione del movimento partigiano, che si affermò durante la guerra civile e che per questi tratti la distingue poco dai modelli della fotografia militare, dipende in gran parte proprio dalla funzione testimoniale della fotografia resistenziale. $\mathrm{Su}$ questo aspetto le esperienze di democrazia e di autogoverno partigiano, nonostante la radicalità e l'originalità che le contraddistinse, incisero scarsamente. In quel frangente, per arrivare a una ridefinizione delle culture visuali ci

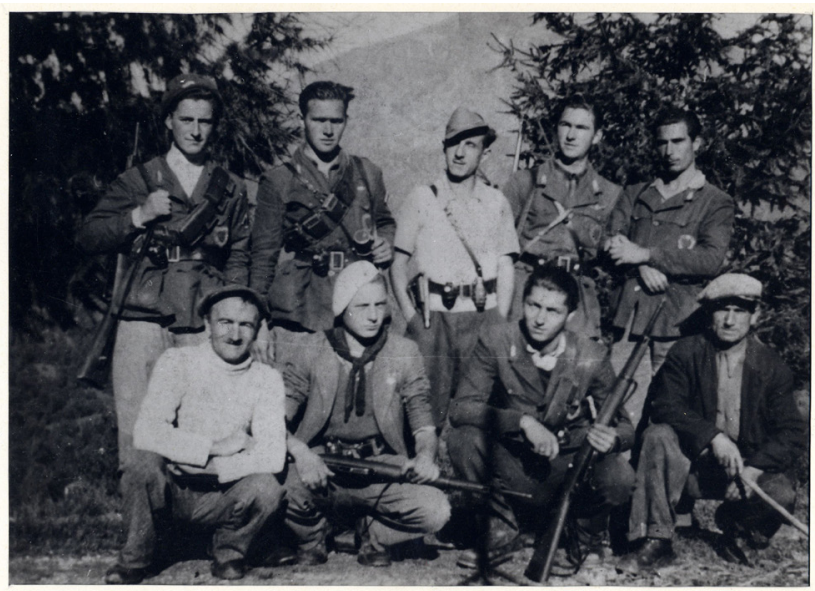

Fig. 13 Gruppo di partigiani a Borgiallo (To) nell'ottobre 1944 [Archivio Istoreto, fondo Novascone] sarebbe stato bisogno di una trasformazione repentina della percezione della guerra e delle aspettative dei vari protagonisti, che non si verificò. Tutti vissero le settimane di libertà consapevoli della fugacità e della provvisorietà della situazione, la portata di quegli eventi fu probabilmen- 


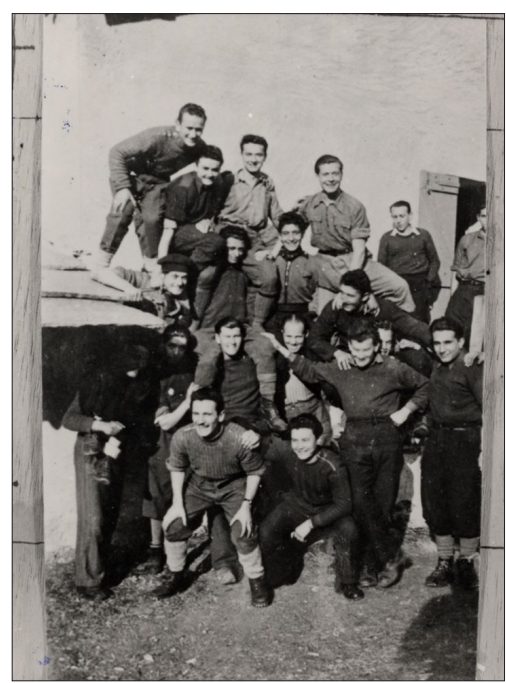

te limitata e la durata troppo breve per dar vita a inedite rappresentazioni della realtà. Proprio per queste ragioni troviamo le stesse fotografie di partigiani nell'estate del ' 44 e nell'inverno successivo, in val Chisone e a Montefiorino.

Fig. 14 Autunno 1943-primavera 1944, gruppo di partigiani della banda Rolandino (Natale Rolando) nelle valli di Lanzo [Centro di documentazione di storia contemporanea e della resistenza nelle Valli di Lanzo Nicola Grosa]

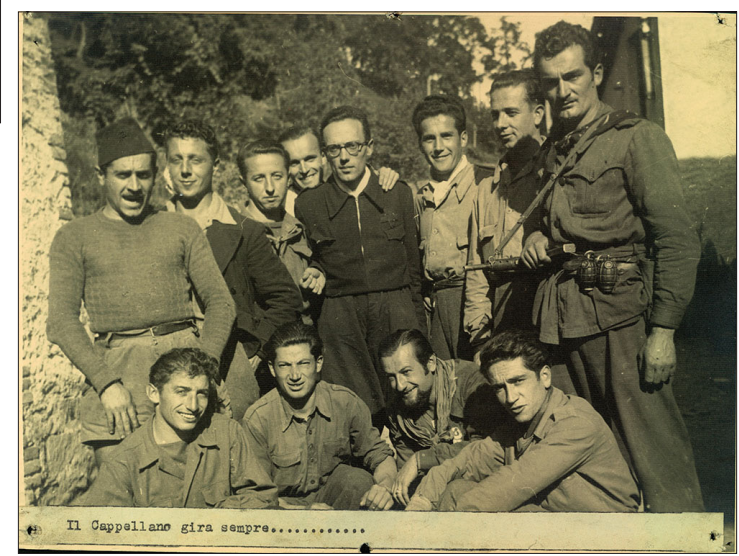

Fig. 15 Settembre 1944. Brigata Vall'Ellero. Prea di Roccaforte [Archivio Istoreto, fondo fotografico Fondazione solidarietà nazionale]

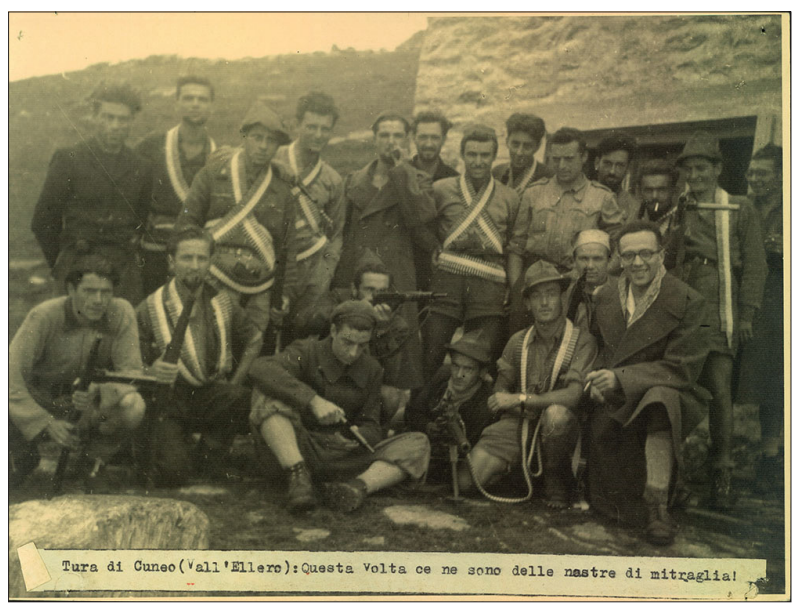

Fig. 16 Tura di Cuneo (Vall'Ellero) [Archivio Istoreto, fondo fotografico Fondazione solidarietà nazionale] 


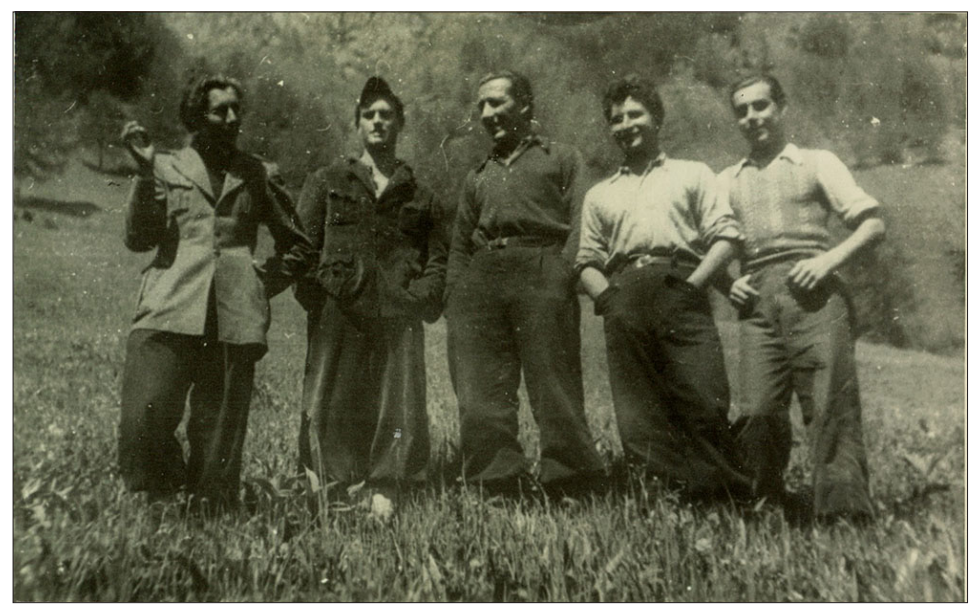

Fig. 17 Maggio

1944. Val

Maira [Archivio

Istoreto, fondo

fotografico

Fondazione

solidarietà na-

zionale]

\section{Guerra e dopoguerra: una memoria marginale tra passato e presente}

Nel tentativo di delineare il quadro preciso entro cui studiare la rappresentazione fotografica della zona libera della val Chisone, mi dedicherò in conclusione a prendere brevemente in esame la seconda ricezione delle immagini, cioè l'uso che ne fu fatto nel dopoguerra. Nell'opera di costituzione degli archivi fotografici della Resistenza, l'analisi della produzione di documenti non può essere mai disgiunta da quella delle iniziative finalizzate alla costruzione della memoria pubblica dell'evento. Le campagne di raccolta delle fotografie promosse nel dopoguerra per divulgare la storia partigiana attraverso le mostre locali, nazionali e internazionali, sono state fondamentali non solo nel creare importanti fondi documentali, ma soprattutto nel consolidare e diffondere una certa immagine della Resistenza italiana, selezionando e favorendo l'acquisizione di materiale fotografico in grado di co-

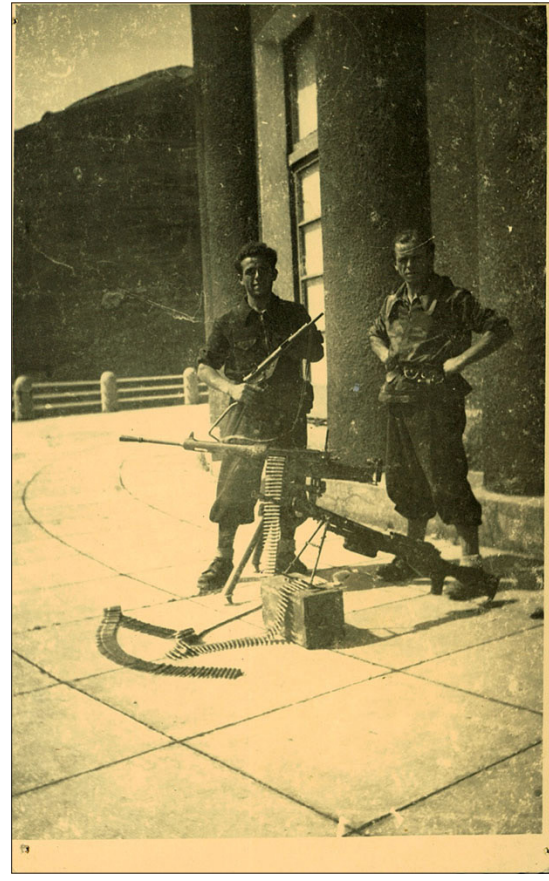

Fig. 18 Partigiani a Sestriere [Archivio Istoreto, fondo fotografico Fondazione solidarietà nazionale] 
municare caratteri ben precisi della guerra di liberazione ${ }^{50}$. In definitiva, bisogna chiedersi se l'operazione di creazione della memoria resistenziale avviata nel dopoguerra abbia influito sulla raffigurazione della lunga estate del 1944, anche a decenni di distanza.

Ho cercato dunque di comprendere quale spazio sia stato dedicato alla rappresentazione fotografica delle zone libere nelle mostre sulla Resistenza allestite nel dopoguerra. Nella prima esposizione piemontese, quella del 1945 realizzata per conto della Fondazione di solidarietà nazionale, vi fu molta enfasi sulle fasi della guerra e sulle vittime partigiane, mentre non pare ci sia stato alcuno spazio per il racconto delle zone libere piemontesi, nonostante prevalesse la documentazione delle Langhe, dove operò la formazione del fotografo De Cavero: molti suoi scatti accompagnarono il racconto, ma di nessuno furono evidenziati eventuali rimandi alla zona liberata ${ }^{51}$. Nessun riferimento sembra esserci neppure nella $\mathrm{Mo}$ stra della Resistenza in Piemonte del 1946, ampliamento della precedente. I temi prevalenti risultano la dimensione militare della Resistenza, i martiri, la tortura e la sofferenza delle vittime, la vita partigiana, la stampa clandestina, la liberazione [Mostra della resistenza in Piemonte 2005, 86-91; Alessandrone Perona 2005, 47-56; D’Arrigo 2014] $]^{52}$. Nella Mostra del risorgimento ossolano (Domodossola, 23 settembre 1945) non poteva mancare una sezione sulla repubblica, che si presenta però senza immagini con soli manifesti e documenti testuali ${ }^{53}$. In sintesi: nelle mostre piemontesi del dopoguerra non comparirono fotografie particolari delle zone libere, anche perché il tema venne in gran parte trascurato. Che cosa accadde invece nelle altre esposizioni in Italia e in quelle allestite dagli italiani in occasione di eventi internazionali? Dagli studi fin qui compiuti non sembra che l'argomento stesse particolarmente a cuore ai vari curatori. Per esempio, i titoli dei pannelli della Mostra della liberazione, la prima del genere realizzata a Mila-

50 Per esempio, a proposito della mostra della liberazione realizzata a Torino già nell'estate del 1945, Carlo Boccazzi Varotto ha scritto: "La prima mostra promossa è quella alla GAM di Torino nell'estate '45, su iniziativa di Felice De Cavero e altri». II materiale fotografico raccolto in Piemonte «rappresenta così uno dei principali patrimoni di immagini cui si è attinto in questi sessant'anni, anche inconsapevolmente, per rappresentare la resistenza italiana» [Boccazzi Varotto 2005, 39]. Adeguatamente integrata e rivista, la mostra fu riallestita nel 1946.

51 Si veda Archivio Istoreto, fondo fotografico Fondazione solidarietà nazionale, per esempio le immagini della sezione "vita partigiana". Cfr. Boccazzi Varotto 2005.

52 Dei contenuti dell'esposizione parlano alcuni documenti in Archivio Istoreto, fondo Istituto storico della Resistenza in Piemonte, busta E ISRP 2, f. 8, Per uno schema di ordinamento della mostra della resistenza; ivi, Elenco materiale mostra della resistenza (7 novembre 1946); Elenco dei documenti sulla resistenza in Piemonte (tratti dalla Mostra della Resistenza, in aggiunta a quelli già direttamente consegnati al dr. Delzell e al dr. Nemirovsky).

53 C'era una foto, quasi certamente scattata dopo la guerra e comunque successiva all'esperienza della repubblica partigiana, raffigurante la sala in cui si radunava la giunta di governo [Mostra del risorgimento ossolano, 2005, 76-79]. 
no ed esposta già nel luglio del 1945, non sembrano fare riferimento a quei fatti [Mignemi, 1995, 46; Solaro, 2005, 27-38]. Nemmeno nelle mostre presentate in Europa vi fu spazio per le multiformi esperienze democratiche, poiché in quei casi prevalse chiaramente la priorità di costruire una diversa identità dell'Italia rispetto alla pesante eredità fascista ${ }^{54}$.

Non solo in Piemonte, dunque, la memoria visuale del periodo delle zone libere non trovò spazio nei principali appuntamenti celebrativi del dopoguerra, almeno in quelli finora studiati. In generale, il racconto della Resistenza fornito dalle mostre marginalizzò, se non escluse del tutto, la storia delle repubbliche partigiane e delle esperienze di autogoverno. Gli argomenti prevalenti in quelle esposizioni furono ben altri: l'organizzazione del movimento partigiano, il Cln, le azioni militari, i caduti e la sofferenza per la tortura, le varie fasi della lotta di liberazione nel quadro più ampio della guerra mondiale, gli altri fenomeni di resistenza in Europa. L'uso pubblico della storia della Resistenza nel dopoguerra, volto soprattutto a tenere viva la memoria della lotta antifascista minacciata dall'oblio, a legittimare i partiti che l'avevano condotta attraverso il coordinamento del Cln e, su un piano internazionale, a emancipare gli italiani sconfitti dal passato fascista, orientò così non solo i temi affrontati dalle mostre, ma anche la correlata ricerca documentaria, improntando la formazione dei primi archivi, anche di quelli fotografici, alle medesime esigenze celebrative ${ }^{55}$.

Si riscontra dunque un'evidente sintonia tra i tratti della Resistenza divulgati dalle mostre e le fotografie partigiane: nel dopoguerra si consolidò un canone fotografico della guerra di liberazione nel quale primeggiò l'immagine militare, mascolina e giovanile della Resistenza, la stessa impressa in moltissimi ritratti individuali o di gruppo già durante il conflitto [Mignemi, 1995] $]^{56}$. In questo canone, durato a lungo, non c'era spazio per altri elementi, dunque nemmeno per raccontare l'atmosfera delle zone libere nel 1944. Anche per effetto dei processi di costruzione della memoria della guerra partigiana attivati a guerra terminata, la memoria visuale delle zone libere si è così omologata al più generale canone fotografico della Resistenza italiana.

Non è possibile sostenere con certezza che non esistano fotografie delle zone li-

54 Qualche riferimento c'è nella mostra di Bordeaux del 1946, ma senza immagini, e comunque i pannelli sulle zone libere riguardano in realtà la rioccupazione del territorio da parte delle truppe nazi-fasciste dopo le esperienze democratiche, per esempio le repubbliche dell'Ossola e di Montefiorino [Mignemi 2005, 111-172].

55 Indagini approfondite su singoli fondi fotografici personali servono a confrontare la memoria ufficiale e quella privata, comparandone anche i tempi di sedimentazione e la stratificazione.

56 Tra gli altri elementi prevalenti del canone fotografico c'era la sofferenza rappresentata dalle vittime partigiane. 
bere con caratteristiche differenti da quelle per ora rilevate, in grado, per esempio, di dare spazio alle pratiche di governo partigiano o di amministrazione democratica: potrebbero essercene in qualche scatolone, ma esse non sono state valorizzate, anzi sono state finora dimenticate. Il risultato finale dunque non cambierebbe. Com'è noto, infatti, la rimozione di fondamentali aspetti della storia della Resistenza ha condizionato per decenni la ricostruzione storica, non favorendo il reperimento di fonti in grado di documentarli: quanto è accaduto per esempio per la partecipazione delle donne, per la guerra senz'armi e per il contributo dei civili, a lungo rimossi dalla memoria collettiva, si è verificato nel caso delle zone libere.

\section{La memoria visuale delle zone libere: storia di un'assenza}

Come si forma la memoria visuale di un evento storico come la nascita delle zone libere nell'Italia del 1944? L'analisi della fotografia della val Chisone ha mostrato che le cause sono di vario tipo: le scelte del fotografo, i caratteri locali della guerra partigiana, la funzione della fotografia in guerra, la circolazione delle immagini, l'uso celebrativo della documentazione fotografica nel dopoguerra. Ho cercato di farle affiorare pensando all'intricata trama di relazioni nella quale le singole fotografie assumono significato: il contenuto simbolico di ogni immagine non è infatti mai dato, ma anzi dipende strettamente dal contesto di produzionericezione. Lo studio di caso dimostra che l'esame delle fotografie partigiane delle zone libere non si può limitare a uno solo dei due aspetti, poiché la molteplicità dei fattori che favorì la formazione della memoria visuale della Resistenza obbliga lo storico a passare continuamente dalla sfera privata a quella pubblica, dalla dimensione individuale a quella collettiva, dallo spazio locale a quello nazionale, dai mesi della guerra al dopoguerra. L'analisi di ciascuno di questi piani offre risposte specifiche, necessarie a capire la complessa rete di interazioni entro cui studiare la fotografia della Resistenza, e conduce a risultati parziali, che vanno posti tra loro in relazione per poter meglio delineare le culture visuali dei protagonisti di quelle vicende. Il mio studio considera ogni contesto sempre collegato agli altri; tutti insieme restituiscono un messaggio piuttosto evidente: scrivere la storia della rappresentazione fotografica della zona libera della val Chisone significa anzitutto fare la storia di un'assenza, di un silenzio, di un'omissione.

Ma nella storia fotografica delle zone libere, la valle in cui operò Ettore Serafino è un'eccezione? L'allargamento dell'osservazione dal singolo caso alla storia delle zone libere ci spinge a generalizzare la domanda iniziale e a chiederci in che modo la memoria visuale della Resistenza italiana, costruita durante e dopo la 
guerra, abbia marginalizzato i brevi periodi di libertà e di governo partigiano del 1944. La ricerca è in corso e le varie ipotesi formulate nel saggio dovranno essere vagliate alla luce di un'analisi più approfondita del fondo fotografico di Ettore Serafino, delle fonti relative alle altre realtà e della cultura fotografica dell'epoca.

\section{Bibliografia}

Alessandrone Perona E. 2005, in Mignemi A. e Solaro G. (eds.) 2005, Un'immagine dell'Italia. Resistenza e ricostruzione. Le mostre del dopoguerra in Europa

Alpers S. 1984, Arte del descrivere: scienza e pittura nel Seicento olandese, Torino: Bollati Boringhieri (ed. or. 1983)

Barthes R. 1980, La camera chiara, Torino: Einaudi (ed. or. 1980)

Battaglia P. e Silingardi C. (eds.) 2004, Obiettivo Montefiorino: le fotografie di Olimpo e Aldo Corti (1910-1975), Modena: RFM Edizioni

Baxandall M. 2000, Forme dell'intenzione. Sulla spiegazione storica dell'opera d'arte, Torino: Einaudi (ed. or. 1985)

Benjamin W. 1966, Piccola storia della fotografia, in id., L'opera d'arte nell'epoca della sua riproducibilità tecnica. Arte e società di massa, Torino: Einaudi (ed. or. 1955)

Boccalatte L., D’Arrigo A. e Maida B. (eds.) 2009, Guida ai luoghi della guerra e della Resistenza nella provincia di Torino, Torino: Blu Edizioni

Boccazzi Varotto C. 2005

Le mostre della Fondazione di Solidarietà Nazionale e dell'esercito 1945-46, in Mignemi A. e Solaro G. (eds.) 2005, Un'immagine dell'Italia. Resistenza e ricostruzione. Le mostre del dopoguerra in Europa

Bourdieu P. (ed.) 1972, La fotografia. Usi e funzioni sociali di un'arte media, Rimini: Guaraldi (ed. or. 1965)

Bravo A. 1965, La repubblica partigiana dell'Alto Monferrato, Torino: Giappichelli

Burke P. 2002, Testimoni oculari. Il significato storico delle immagini, Roma: Carocci (ed. or. 2001)

Buvoli A. 2013, La repubblica partigiana della Carnia e dell'Alto Friuli, Bologna: il Mulino

Buvoli A. e Nigris C. 2004, Percorsi della memoria civile. La Carnia, la Resistenza, Udine: Istituto friulano per la storia del movimento di liberazione

Castelnuovo E. 1985, Arte, industria, rivoluzioni. Temi di storia sociale dell'arte, Torino: Einaudi

Castelnuovo E. e Ginzburg C. 1979, Centro e periferia, in Storia dell'arte italiana. Questioni e metodi, Torino: Einaudi 
Clarke G. 2009, La fotografia. Una storia culturale e visuale, Torino: Einaudi (ed. or. 1997)

D'Arrigo A. 2014, Genesi di un patrimonio: l'archivio fotografico dell'Istituto storico della Resistenza in Piemonte, in Tabor D. (ed.) 2014, Le ragioni di una scelta nelle fotografie della Resistenza

D'Autilia G. 2005, L'indizio e la prova. La storia nella fotografia, Milano: Bruno Mondadori

De Luna G. 1993, L'occhio e l'orecchio dello storico. Le fonti audiovisive nella ricerca e nella didattica della storia, Firenze: La Nuova Italia

De Luna G. e Mignemi A. (eds.) 1997, Storia fotografica della Repubblica sociale italiana, Torino: Bollati Boringhieri

Del Boca A. (ed.) 2004, La “Repubblica” partigiana dell'Ossola, Crodo: Centro Studi Piero Gnocchi

Evans J. e Hall S. (eds.) 1999, Visual Culture. The Reader, London: Sage

Fotografia e stile 1978, "Progresso fotografico", LXXXV, 2

Frassati F. (ed.) 1984, La Repubblica dell'Ossola, Domodossola: Ambiente

Freedberg D. 1993, Il potere delle immagini. Il mondo delle figure: reazioni ed emozioni del pubblico, Torino: Einaudi (ed. or. 1989)

Freund G. 1976, Fotografia e società. Riflessione teorica ed esperienza pratica di una allieva di Adorno, Torino: Einaudi (ed. or. 1974)

Gay Rochat D. 1985, La Resistenza nelle valli valdesi, Torino: Claudiana

Gilardi A. 1976, Storia sociale della fotografia, Milano: Feltrinelli

Ginzburg M. 2004, Indagini su Piero. Il Battesimo, il ciclo di Arezzo, la Flagellazione di Urbino, Torino: Einaudi

Giovannini Luca A. 2014, Fotografare per diletto: appunti e buone pratiche per una storia della fotografia amatoriale, in Tabor D. (ed.) 2014, Le ragioni di una scelta nelle fotografie della Resistenza

Giovannini Luca A. e Pierobon A. (eds.), 2014, Per una storia sociale dell'arte: bilanci, esperienze, prospettive. Intervista a Enrico Castelnuovo, "Contesti. Rivista di microstoria", 1

Gombrich E.H. 1965, Arte e illusione. Studio sulla psicologia della rappresentazione pittorica, Torino: Einaudi (ed. or. 1960)

Haskell F. 1989, Le metamorfosi del gusto: studi su arte e pubblico nel $18^{\circ}$ e $19^{\circ}$ secolo, Torino: Bollati Boringhieri

Krauss R. 1999, Photography's Discoursive Spaces, in Evans J. e Hall S. (eds.) 1999, Visual Culture. The Reader, London: Sage

La Repubblica prima della Repubblica. Val d'Ossola 1944: democrazia repubblicana alla prova 2011, Napoli: Edizioni scientifiche italiane 
La stampa ed i mezzi di comunicazione dei partigiani e della Repubblica dell'Ossola 2006, Domodossola: Anpi

Lenzotti S. 2009, La zona libera di Montefiorino, Modena: Artestampa

Linguaggio e fotografia 1977, "Progresso fotografico", LXXXIV, 12

Lusini S. 1996 (ed.), Fototeche e archivi fotografici. Prospettive di sviluppo e indagini delle raccolte, Prato: Comune di Prato

Marcellin M. 1966, Alpini... finché le gambe vi portano, Pinerolo (To): Scuola Tipografica dei Padri Giuseppini

Mignemi A. 1995, Storia fotografica della Resistenza, Torino: Bollati Boringhieri

Mignemi A. 2000, Immagini di Resistenza: storia, memoria, fotografia, Chiusa Pesio (Cn): Comune di Chiusa Pesio

Mignemi A. 2003, Lo sguardo e l'immagine. La fotografia come documento storico, Torino: Bollati Boringhieri

Mignemi A. 2005, La mostra ritrovata, in Mignemi A. e Solaro G. (eds.) 2005, Un 'immagine dell'Italia. Resistenza e ricostruzione. Le mostre del dopoguerra in Europa

Mignemi A. e Solaro G. (eds.) 2005, Un'immagine dell'Italia. Resistenza e ricostruzione. Le mostre del dopoguerra in Europa, Milano: Skira

Mostra del risorgimento ossolano 2005, in Mignemi A. e Solaro G. (eds.) 2005, Un'immagine dell'Italia. Resistenza e ricostruzione. Le mostre del dopoguerra in Europa

Mostra della resistenza in Piemonte 2005, in Mignemi A. e Solaro G. (eds.) 2005, Un 'immagine dell'Italia. Resistenza e ricostruzione. Le mostre del dopoguerra in Europa

Noi Alpini della Val Chisone 1945, Torino: Impronta

Obert F. 1993, Diario inedito di Franco Obert, partigiano a Cumiana e in Val Chisone, "Eco del Chisone", 22 aprile

Oliva E. 1983, La Repubblica partigiana dell'Ossola e altri episodi della guerra di Liberazione, Domodossola: Libreria Grossi

Ortoleva G. 1983, La fotografia, in Il Mondo contemporaneo, vol. X Gli strumenti della ricerca, Firenze: La Nuova Italia

Quarenghi G. (ed.) 2003, Un romanzo familiare: la fotografia come memoria individuale e collettiva, Vilminore di Sclave: Il filo di Arianna

Remaggi A., Silingardi C. e Teodoro F. 1994, Le montagne della libertà. Immagini per la storia della Repubblica partigiana di Montefiorino, Montefiorino: Comune di Montefiorino

Rostan G. 2003, Tempi di guerra. Diario partigiano in val Chisone e Germanasca, LAReditore

Sega M.T. 1988, La storia scritta con la luce, "I viaggi di Erodo", I

Serafino E. 1945, Dalla guerriglia alla guerra in Noi Alpini della Val Chisone 1945

Serafino E. 1994, La libera Repubblica della val Chisone, "Eco del Chisone", 16 giugno 
Serafino E. 2001, Quando il vento le pagine sfoglia, Collegno (To): Roberto Chiaramonte Editore

Silingardi C. 2005, Museo della Repubblica partigiana di Montefiorino, Modena: Artestampa

Solaro G. 2005, Le mostre fotografiche della resistenza nei documenti dell'Istituto nazionale per la storia del movimento di liberazione in Italia, in Mignemi A. e Solaro G. (eds.) 2005, Un'immagine dell'Italia. Resistenza e ricostruzione. Le mostre del dopoguerra in Europa

Sontag S. 1978, Sulla fotografia. Realtà e immagine nella nostra società, Torino: Einaudi (ed. or. 1973)

Sperber D. e Wilson D. 1993, La pertinenza, Milano: Anabasi (ed. or. 1986)

Tabor D. (ed.) 2014, Le ragioni di una scelta nelle fotografie della Resistenza, Torino: Seb 27

Trabucco A. 1984, Resistenza in val Chisone e nel pinerolese, Pinerolo (To): Arti Grafiche

Vallauri C. (ed.) 2013, Le repubbliche partigiane: esperienze di autogoverno democratico, Roma-Bari: Laterza 2013

Vottero Fin T. 1994, Resistenza partigiana nelle valli di Lanzo, nel Canavese e in val di Susa. Immagini e storia della lotta per la libertà, Torino: Edizioni CDA

Zannier I. 1978, 70 anni di fotografia in Italia, Modena: Punto e virgola

Zannier I. 1986, Storia della fotografia italiana, Roma: Laterza

\section{Risorse}

Istoreto - Intervista a Ettore Serafino, Pinerolo 10 settembre 2008 http://www.metarchivi.it/dett_documento.asp?id=11391\&tipo=VIDEO

Archivio Corti Olimpio e Aldo - Raccolte fotografiche modenesi http://raccoltafoto.comune.modena.it/index.html 